\title{
Febrile infection-related epilepsy syndrome (FIRES): prevalence, impact and management strategies
}

REVIEW

\author{
Domenico Serino' \\ Marta Elena Santarone ${ }^{2}$ \\ Davide Caputo ${ }^{3}$ \\ Lucia Fusco 2 \\ 'Department of Child Neurology, Royal \\ Aberdeen Children's Hospital, Aberdeen, \\ UK; ${ }^{2}$ Department of Neuroscience, \\ Bambino Gesù Children's Hospital, \\ Rome, Italy; ${ }^{3}$ Neurology Unit, \\ Neuroscience Department, San Paolo \\ Hospital, Milan, Italy
}

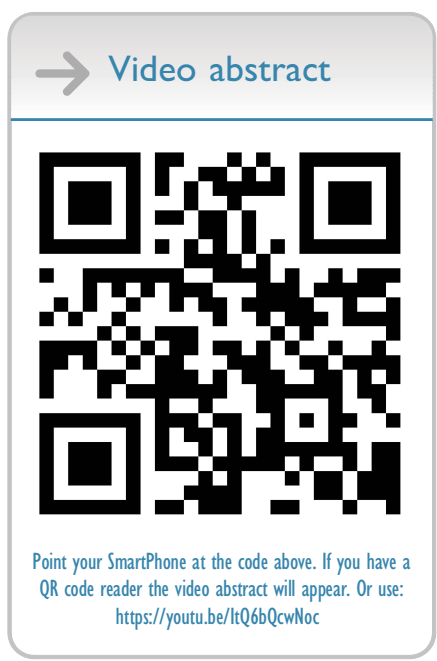

Correspondence: Lucia Fusco Bambino Gesù Children's Hospital, Piazza S. Onofrio 4, 00165 Rome, Italy Tel +393356163494

Email lucia.fusco@opbg.net
This article was published in the following Dove Press journal:

Neuropsychiatric Disease and Treatment

\begin{abstract}
Febrile infection-related epilepsy syndrome (FIRES) is a rare catastrophic epileptic encephalopathy with a yet undefined etiology, affecting healthy children. It is characterized by acute manifestation of recurrent seizures or refractory status epilepticus preceded by febrile illness, but without evidence of infectious encephalitis. To date, the absence of specific biomarkers poses a significant diagnostic challenge; nonetheless, early diagnosis is very important for optimal management. FIRES is mostly irreversible and its sequelae include drug-resistant epilepsy and neuropsychological impairments. The treatment of FIRES represents a significant challenge for clinicians and is associated with low success rates. Early introduction of ketogenic diet seems to represent the most effective and promising treatment. This review aims to highlight the most recent insights on clinical features, terminology, epidemiology, pathogenesis, diagnostic challenges and therapeutic options.
\end{abstract}

Keywords: FIRES, NORSE, epilepsy syndromes, inflammatory epilepsies, status epilepticus, drug-resistance

\section{Introduction}

The acronym FIRES stands for "Febrile Infection Related Epilepsy Syndrome" and was used for the first time by van Baalen and colleagues in $2010,{ }^{1}$ although the same clinical entity has been called in varying ways by different authors. ${ }^{23}$

The term refers to a rare catastrophic epileptic encephalopathy with a yet undefined etiology, characterized by acute manifestation of recurrent seizures or refractory status epilepticus (SE) preceding febrile illness, but without evidence of infectious encephalitis. Being mostly irreversible, FIRES is often followed by the development of drugresistant epilepsy and neuropsychological impairments occurring without latency. It is sporadic and extremely rare, which significantly hampers the collection of clinical data necessary to develop effective management strategies. To date, the absence of specific biomarkers poses a significant diagnostic challenge; nonetheless, early diagnosis is very important for optimal management. Diagnostic and therapeutic delay contributes to poor outcome in SE, and although seizure control is often achieved only once the underlying cause is identified, over 200 uncommon disorders may be responsible for SE. ${ }^{4}$ In up to $60 \%$ of cases of de novo refractory SE, diagnostic workup fails to reveal the underlying etiology, ${ }^{5,6}$ which is the typical and problematic clinical scenario that FIRES entails. We hope that this clinical review may help in correctly recognizing and managing FIRES among this group of small but not negligible patients. 


\section{Terminology}

The most recent definition of FIRES comes from the proceedings of the First International new onset status epilepticus (NORSE) and FIRES Symposium. ${ }^{7}$ The proposed consensus definition identifies FIRES as a subcategory of NORSE. This acronym was coined by Wilder-Smith and colleagues ${ }^{8}$ to describe a form of new onset refractory SE in patients without active epilepsy or other preexisting relevant neurological disorders, without a clear acute or active structural, toxic or metabolic cause. Initially, NORSE was considered as specific of the adult population while FIRES was specific of the pediatric population; however, this distinction has been recently challenged since it does not seem to be based on strong scientific evidence. ${ }^{7}$ In this light, FIRES is defined as a subtype of NORSE that requires a prior febrile infection, with fever starting between 2 weeks and $24 \mathrm{hrs}$ prior to onset of refractory SE, with or without fever at onset of SE. This applies to all ages and there may or may not be fever at the time of onset of SE. ${ }^{7}$ This definition excludes prolonged febrile seizures, as they usually occur in children who have onset of fever $<24 \mathrm{hrs}$ prior to onset of seizures or whose fever is recognized only after onset of seizures. ${ }^{9}$

\section{Epidemiology}

Refractory status epilepticus following a common febrile episode in the absence of identified infectious agents is not frequently reported; therefore, it is extremely difficult to extract epidemiological data from available literature. In addition to this, before the term FIRES was adopted, cases of encephalitis of unknown or presumed immune etiology have been described with many different terms, such as "acute encephalitis with refractory, repetitive partial seizures", "severe refractory status epilepticus due to presumed encephalitis", "idiopathic catastrophic epileptic encephalopathy", "devastating epileptic encephalopathy in school-aged children"12 and "Childhood refractory focal epilepsy following acute febrile encephalopathy". ${ }^{3}$ Affected patients are mainly children between 5 and 13 years of age, with a peak during school age and a male preponderance. ${ }^{13,14}$ Based on a prospective hospital-based German-wide surveillance program, van Baalen and colleagues estimated the annual incidence and prevalence of FIRES among children and adolescents in Germany to be, respectively, 1: 1,000,000 and 1: 100,000. ${ }^{1}$ As mentioned earlier, however, the current consensus definition of FIRES is not limited to childhood and adolescence. The outcome of FIRES is poor, with a death rate of up to
$30 \%,{ }^{1,14}$ refractory epilepsy at follow-up often immediately following the acute phase ${ }^{2}$ and cognitive delay in $66-100 \%$ of the survivors. ${ }^{12,14}$ The survivors with previous normal cognitive levels usually develop learning disabilities, ${ }^{12,14}$ and only a minority survives the episode without any neurologic sequelae. ${ }^{14}$

\section{Pathogenesis}

The pathogenesis underlying FIRES is still a matter of debate. Many different pathogenetic cascades and mechanisms have been hypothesized. Several authors explored the possibility of FIRES being a form of severe infectious encephalitis. ${ }^{10,14}$ The idea was suggested by the typical evolution of FIRES, which follows a febrile event and in most patients is associated with pleyocytosis in the cerebrospinal fluid (CSF). In such light, in a case series of 77 patients, Kramer et al carried out a thorough and extensive investigation for possible infectious agents but found none. ${ }^{14}$ This is not surprising, considering that so far, no published data regarding brain biopsies of FIRES patients have yielded typical findings of encephalitis. ${ }^{1}$ Other clinical elements also seem to diverge from this hypothesis. First of all, seizure control during the acute phase of FIRES is significantly more difficult than in encephalitis while the risk of postencephalitic epilepsy is significantly higher $(0-33 \%$ vs nearly $100 \%) .{ }^{15}$ Secondly, while onset of post-encephalitic epilepsy is typically delayed, in FIRES patients, there is no latency between SE and epilepsy onset. Lastly, the typical biphasic clinical course of FIRES seems more suggestive of an "infection-triggered" process than an infectious disease. Inflammation seems to play an important but not exclusive role in the development of FIRES. As just said, a biphasic clinical course is suggestive of an immune disorder triggered by an infection, even though fever itself does not seem to be a causative agent. Consistently, while febrile SE is almost concomitant with fever insurgence, SE in FIRES begins days after fever onset and said fever is often mild. In this light, inflammation does not seem to trigger an acute event, but more to contribute to the FIRES cascade through a generalized and ongoing lowering of seizure threshold. This might be accomplished by an infection-induced gradual build-up of systemic and brain-born inflammatory cytokines. ${ }^{16,17}$ The slow progressive nature of such a process might be the reason for the delay in FIRES onset. The neuro-inflammatory response, which plays a role in epileptogenesis, seems to be a direct consequence of innate immunity activation which takes place in glial cells, neurons and the blood-brain barrier. ${ }^{18-21}$ Such activation is usually self-limited; however, intrinsic factors such as a genetic predisposition may be responsible for a 
lack of efficient resolution of the process. Progressive accumulation of inflammatory cytokines could then give way to a vicious cycle of aberrant hyperexcitability ${ }^{18}$ and/or a structural epileptogenic remodeling of brain networks. ${ }^{22}$ Several other potential causes have been suspected and discussed in the literature, but currently, none of them has been confirmed. The abovementioned biphasic timing of FIRES has led researchers to look for an autoimmune mechanism; however, the search for specific autoantibodies has so far yielded scarce results. $^{14,23}$ One notable exception is the reported finding of anti-GABA A Receptor antibodies in a case of FIRES which responded to ketamine and high-dose intravenous steroids. ${ }^{24}$ Response to immunotherapy, however, seems to be the exception, not the rule. ${ }^{23}$ An autoimmune reaction may, however, have a role in at least some cases of FIRES in which it may act as a trigger for a metabolic disease or channelopathy. ${ }^{25-27}$ Some case series reports showed no clear correlation between FIRES and SCN1A, PCDH19 or POLG1 gene mutations. ${ }^{28,29}$ In conclusion, however, the underlying pathogenic mechanism of FIRES seems to be a double-hit process: a synergistic effect between an immune response to a febrile illness or to an infection affecting the brain and an intrinsic predisposition toward an auto-sustaining epileptogenic process. We illustrated a possible mechanistic model of epileptogenesis in FIRES in Figure 1.

\section{Diagnosis}

As no certain cause of FIRES has been identified yet in the majority of patients, diagnosis of FIRES is essentially clinical $^{7}$ and no biological markers or genetic testing are available for diagnosis. Affected patients have an unremarkable neurological history and, in close relation with a febrile illness (mainly upper respiratory tract infection or, less frequently, gastroenteritis), abruptly develop several seizures (even 50-100 per day). In less than 24 hours, seizures evolve toward a prolonged, refractory SE. Seizures and SE dominate the clinical presentation, and in more than $50 \%$ of patients, fever has disappeared when the first seizures occur. ${ }^{1}$ Cognitive and behavioral prodromes are unlikely and may suggest an alternative diagnosis (i.e. autoimmune encephalitis). ${ }^{30,31}$ Seizures at disease onset are mainly focal or secondarily generalized. ${ }^{14}$ The most frequent ictal

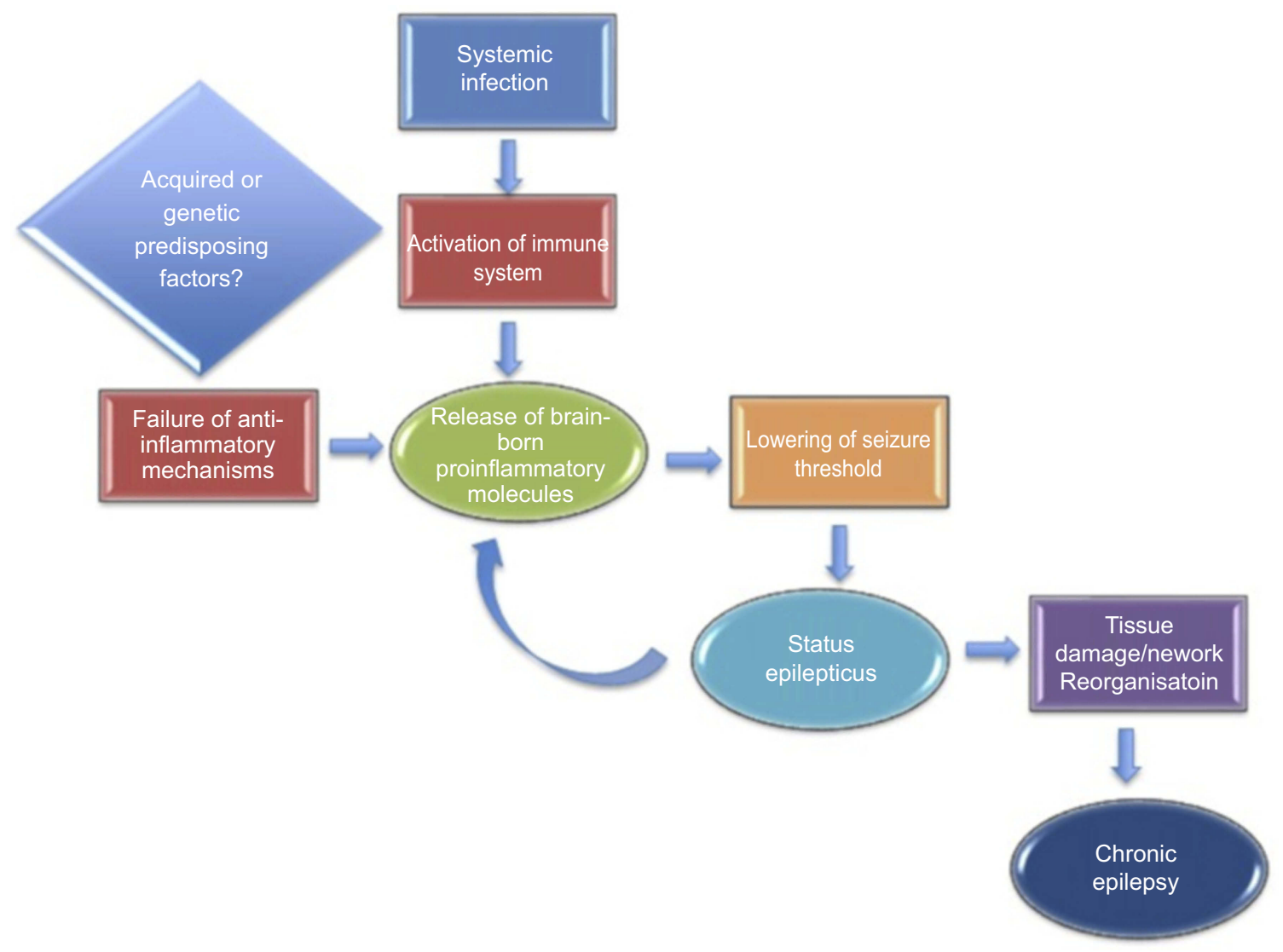

Figure I A possible mechanistic model of epileptogenesis in FIRES: a systemic infection acts as a trigger of the "neuroinflammatory cascade", creating a vicious circle leading to chronic epilepsy. 
manifestations reported ${ }^{30}$ include chewing movements, lateral head deviation and subtle vegetative signs (i.e. modification of heart rate and/or mydriasis), though clonic jerks of the mouth with drooling and jerks of the upper or lower limbs can also occur. Between seizures, patients are drowsy or even comatose, requiring high-dose anesthetics or midazolam and mechanical ventilation. SE is usually prolonged and lasts several weeks or months despite many antiepileptic drug (AED) trials. ${ }^{12,14}$ In almost all cases, the outcome is characterized by chronic intractable epilepsy and major cognitive disabilities. ${ }^{14}$ By definition, FIRES occurs as a fever-triggered NORSE in patients without preceding epilepsy or other neurological disorders without clear or active causes. ${ }^{5}$ Therefore, the initial clinical assessment is crucial and extensive investigations are necessary to exclude similar conditions associated with acute onset of SE and in particular those amenable to specific treatment. Careful history taking, neurological examination and basic laboratory testing might identify the most common etiologies of SE, such as discontinuation or changes in AED regimen, intoxication, central nervous system infections, acute or remote structural brain injury and acute metabolic imbalances. ${ }^{7}$ CSF testing is usually negative, with moderate to no pleocytosis and absence of markers for viral infections and autoimmunity (oligoclonal band) ${ }^{30}$ as well as antineural antibodies (mainly anti-VGCK complex, anti-NMDA Receptor, anti-AMPA Receptor, and anti-GABA B Receptor). ${ }^{32}$ During the acute phase, standard EEG and continuous EEG monitoring are of paramount importance in guiding correct therapeutic interventions, assessing sedation levels and recognizing non-convulsive SE. ${ }^{33}$ Although seizures are often generalized, their semiology indicates focal onset and EEG ictal recordings confirm that discharges are often temporal or perisylvian with opercular extension. Interestingly, in a series published by Van Baalen et al., ictal and interictal EEG data showed a single epileptic focus in less than $10 \%$ of patients. ${ }^{32}$ The frequent presence of a shifting focal seizure pattern indicates that both hemispheres are affected while interictal EEG recordings show generalized slow waves. ${ }^{30}$ Unfortunately, EEG has a limited value in differentiating FIRES from epilepsy or encephalopathy of possible autoimmune etiology. Initial brain MRI scans are mostly negative or may show abnormalities predominantly in the temporal regions, or additionally, in the insula and the basal ganglia, that may be both transient or irreversible. ${ }^{1,10,12}$ Early MRI scans (within the first weeks) may show swelling of the mesial temporal structures with increased T2-weighted signal as observed in limbic encephalitis. ${ }^{1,30}$ Follow-up MRI scans (after 6 months) may show bilateral mesial temporal atrophy and increased T2-weighted signal but are normal in half of the cases. ${ }^{30}$ Positron emission tomography scans usually highlight large areas of hypometabolism involving the orbitofrontal and temporoparietal cortices bilaterally. There is a good correspondence with the cortical areas associated with language, behavior and memory, which are also the most frequently recorded ictal onset zones. Other evaluations should be undertaken based on the case's characteristics. It is important to consider that during childhood, many peculiar conditions can result in prolonged $\mathrm{SE}$, including febrile seizures and febrile SE. Also, genetic disorders such as Dravet syndrome and Alpers syndrome involve fever-associated SE. Therefore, a differential diagnosis with genetic associated with fever-induced epilepsy (such as in SCN1A, PCDH19 or POLG1 related epilepsies) should be taken into account.

\section{Treatment}

The treatment of FIRES represents a significant challenge for clinicians and is associated with low success rates, especially during the acute phase. Systematic studies are still lacking and the limited number of published case series propose several therapeutic approaches, none of which appear to be significantly effective, with the exception of the ketogenic diet. In relation to the previously discussed role of inflammation in the pathogenesis of FIRES, high-dose intravenous steroids have been tried despite the risk of severe adverse events. ${ }^{34}$ First-line and second-line immunotherapy might be useful, such as intravenous immunoglobulins and plasmapheresis although efficacy is still debated. The efficacy of tacrolimus, rituximab and/or cyclophosphamide is unclear, even though there are some reports of improvement in patients for which other immunotherapies failed. ${ }^{32-37}$ First-line treatment of SE initially consists of benzodiazepines (lorazepam, diazepam, midazolam, clonazepam), followed by standard anticonvulsant drugs, ${ }^{14}$ although FIRES typically does not respond to high doses of conventional anticonvulsant medications. Second-line intervention usually entails the use of anesthetic agents, in particular, infusion of midazolam and barbiturates (pentobarbital and thiopental). ${ }^{38}$ Midazolam appears as effective as thiopental but is associated with fewer adverse events, faster discharge from intensive care, and better long-term neurological outcome. ${ }^{39,40}$ High doses of phenobarbital have also been proven effective and with fewer side effects than anesthetics. $^{23}$ Further anesthetic agents such as propofol are used in the management of refractory SE, though its 
prolonged use in children is discouraged due to the risk of propofol infusion-related syndrome. ${ }^{34}$ Burst-suppression coma induction is standard care in super-refractory SE, although when barbiturates are weaned seizures frequently reappear. ${ }^{23}$ Moreover, there are concerns regarding its use in FIRES, since prolonged burst-suppression coma has a significant association with a worse cognitive outcome and with a more severe course of the disease. ${ }^{23}$ The ketogenic diet (KD) has been used successfully in patients with refractory seizures and, according to some reports, has shown some efficacy in FIRES. ${ }^{41-43}$ It has been hypothesized that the KD may not only have an anticonvulsant effect (e.g., through the production of decanoic acid which induces a direct inhibition of the post-synaptic excitatory AMPA) but also anti-inflammatory. This might be of great significance in the treatment of FIRES. Early introduction of a KD could be effective not only during the acute phase but also in long-term epilepsy management. ${ }^{23}$ According to some reports, it appears to have a positive effect on both seizure control and cognitive outcome and should therefore be considered precociously in the course of treatment, even as first-line therapy. ${ }^{23}$ Therapeutic hypothermia may be effective in the control of SE by reducing pro-inflammatory cytokine levels and protecting the integrity of the BBB. ${ }^{7}$ Cannabidiol (CBD) acts by decreasing glutamate and gamma-aminobutyric acid synaptic transmission in the brain. In a recently published case series, CBD appeared effective in reducing frequency and duration of seizures in patients with FIRES who had not responded to standard antiepileptic drugs or other therapies. ${ }^{44}$ Anakinra, a recombinant and modified version of the human interleukin-1 receptor antagonist protein, has been used effectively in these patients, both in the acute phase and in the outcome. ${ }^{45}$ Finally, there are isolated reports of good seizure control in FIRES after continuous intravenous administration of magnesium sulfate. ${ }^{46}$ Currently, no systematic studies on the treatment of the aftermath of FIRES exist. The existing data collected from a limited number of reports show that seizures are mostly very difficult to treat and often require polytherapy. Vagus nerve stimulation (VNS) is generally introduced as chronic treatment for seizure control after recovery from SE. Notwithstanding, VNS appears to have a favorable effect on SE, NORSE and generalized convulsive seizures in children with post-FIRES medically intractable epilepsy. ${ }^{47,48}$ For the latter, the use of CBD has also been proposed ${ }^{49,50}$ We have summarized the most commonly used and effective. treatments of FIRES in Table 1.
Table I Treatment strategies in FIRES

\begin{tabular}{|c|c|}
\hline Treatment & Range of dosages \\
\hline Ketogenic diet & $\mathrm{I}: \mathrm{I}$ to $4: I$ Ketogenic ratio ${ }^{23}$ \\
\hline Cannabidiol & $15-25 \mathrm{mg} / \mathrm{kg} / \mathrm{day}^{44}$ \\
\hline Midazolam & Bolus $0.03-0.5 \mathrm{mg} / \mathrm{kg}$ Infusion: $0.02-1 / 2 \mathrm{mg} / \mathrm{kg} / \mathrm{h}^{39}$ \\
\hline Phenobarbital & Infusion $>10 \mathrm{mg} / \mathrm{kg} / \mathrm{h}$ with serum levels $60-100 \mathrm{mg} / \mathrm{dL}^{34}$ \\
\hline Propofol & Bolus $\mathrm{I}-3 \mathrm{mg} / \mathrm{kg}$ Infusion: $0.1-24 \mathrm{mg} / \mathrm{kg} / \mathrm{h}^{34,5 \mathrm{I}}$ \\
\hline Thiopental & Bolus $4-5 \mathrm{mg} / \mathrm{kg}$ Infusion $0.5-12 \mathrm{mg} / \mathrm{kg} / \mathrm{h}^{34}$ \\
\hline Steroids & $\begin{array}{l}\text { Intravenous methylprednisolone } 10-30 \mathrm{mg} / \mathrm{kg} / \mathrm{day} \text { for } \\
3-5 \text { days, then oral prednisone } 1 \mathrm{mg} / \mathrm{kg} / \mathrm{day}^{7}\end{array}$ \\
\hline Plasmapheresis & $3-5$ exchanges, one every other day ${ }^{7}$ \\
\hline Immunoglobulin & Intravenous immunoglobulins $0.4 \mathrm{~g} / \mathrm{kg} /$ day for 5 days $\mathrm{s}^{52,53}$ \\
\hline
\end{tabular}

\section{Conclusions}

FIRES is a yet poorly understood clinical entity, affecting healthy children, with catastrophic sequelae. Treatments are poorly effective and therefore new insights concerning its pathogenesis are still strongly needed in order to develop more targeted therapies.

\section{Disclosure}

All of the authors declare that they have no conflicts of interest in this work.

\section{References}

1. van Baalen A, Häusler M, Boor R, et al. Febrile infection-related epilepsy syndrome (FIRES): a nonencephalitic encephalopathy in childhood. Epilepsia. 2010;51(7):1323-1328. doi:10.1111/j.1528-1167.2010.02535.x

2. Sakuma H, Awaya Y, Shiomi M, et al. Acute encephalitis with refractory, repetitive partial seizures (AERRPS): a peculiar form of childhood encephalitis. Acta Neurol Scand. 2010;121(4):251-2562. doi:10.1111/j.1600-0404.2009.01198.x

3. Specchio N, Fusco L, Claps D, et al. Childhood refractory focal epilepsy following acute febrile encephalopathy. Eur J Neurol. 2011;18(7):952-961. doi:10.1111/j.1468-1331.2010.03253.x

4. Tan RY, Neligan A, Shorvon SD. The uncommon causes of status epilepticus: a systematic review. Epilepsy Res. 2010;91(2-3):111-122. doi:10.1016/j.eplepsyres.2010.07.015

5. Hirsch LJ, Gaspard N. Status epilepticus. Continuum (Minneap Minn). 2013;19(3Epilepsy):767-794. Review. doi:10.1212/01. CON.0000431395.16229.5a

6. Jayalakshmi S, Vooturi S, Sahu S, Yada PK, Mohandas S. Causes and outcomes of new onset status epilepticus and predictors of refractoriness to therapy. J Clin Neurosci. 2016;26:89-94. doi:10.1016/j.jocn.2015.06.032

7. Gaspard N, Hirsch LJ, Sculier C, et al. New-onset refractory status epilepticus (NORSE) and febrile infection - related epilepsy syndrome (FIRES): state of the art and perspectives. Epilepsia. 2018;59(4):745752. doi:10.1111/epi.14022 
8. Wilder-Smith EP, Lim EC, Teoh HL, et al. The NORSE (new-onset refractory status epilepticus) syndrome: defining a disease entity. Ann Acad Med Singapore. 2005;34(7):417-420.

9. Kanemura H, Mizorogi S, Aoyagi K, Sugita K, Aihara M. EEG characteristics predict subsequent epilepsy in children with febrile seizure. Brain Dev. 2012;34(4):302-307. doi:10.1016/j.braindev.2011.07.007

10. Sahin M, Menache CC, Holmes GL, Riviello JJ. Outcome of severe refractory status epilepticus in children. Epilepsia. 2001;42(11):14611467.

11. Baxter P, Clarke A, Cross H, et al. Idiopathic catastrophic epileptic encephalopathy presenting with acute onset intractable status. Seizure. 2003;12(6):379-387.

12. Mikaeloff Y, Jambaqué I, Hertz-Pannier L, et al. Devastating epileptic encephalopathy in school-aged children (DESC): a pseudo encephalitis. Epilepsy Res. 2006;69(1):67-793. doi:10.1016/j.eplepsyres.2006.01.002

13. Nabbout R. STATUS EPILEPTICUS 2013 FIRES and IHHE : delineation of the syndromes febrile Infection - related. Epilepsia. 2013;54:54-56. doi:10.1111/epi.12278

14. Kramer U, Chi C, Lin K, et al. Febrile infection - related epilepsy syndrome (FIRES): pathogenesis, treatment, and outcome. A multicenter study on 77 children. Epilepsia. 2011;52:1956-1965. doi:10.1111/j.1528-1167.2011.03250.x

15. Pillai SC, Mohammad SS, Hacohen Y, et al. Postencephalitic epilepsy and drug-resistant epilepsy after infectious and antibody-associated encephalitis in childhood: clinical and etiologic risk factors. Epilepsia. 2016;57(1):e7-e11. doi:10.1111/epi.13253

16. Vezzani A. Fetal brain inflammation may prime hyperexcitability and behavioral dysfunction later in life. Ann Neurol. 20131;74:1-3. doi:10.1002/ana.23930

17. Vezzani A, Viviani B. Neuromodulatory properties of inflammatory cytokines and their impact on neuronal excitability. Neuropharmacology. 2015;96:70-82. doi:10.1016/j.neuropharm.2014.10.027

18. Nabbout R, Vezzani A, Dulac O, Chiron C, Negri M. Acute encephalopathy with infl ammation-mediated status epilepticus. Lancet Neurol. 2011;10:99-108. doi:10.1016/S1474-4422(10)70214-3

19. Vezzani A, Bartfai T, Bianchi M, Rossetti C, French J. Therapeutic potential of new antiinflammatory drugs. Epilepsia. 2011;52(Supp1 8):67-69. doi:10.1111/j.1528-1167.2011.03242.x

20. Miskin C, Hasbani DM. Status epilepticus: immunologic and inflammatory mechanisms. Semin Pediatr Neurol. 2014;21(3):221-225. doi:10.1016/j.spen.2014.09.001

21. Varadkar S, Cross JH. Rasmussen syndrome and other inflammatory epilepsies. Semin Neurol. 2015;35(3):259-268. doi:10.1055/s-00351552921

22. Reschke CR, Henshall DC. microRNA and Epilepsy. Adv Exp Med Biol. 2015;888:41-70. doi:10.1007/978-3-319-22671-2 4

23. van Baalen A, Vezzani A, Häusler M, Kluger G. Febrile infection - related epilepsy syndrome : clinical review and hypotheses of epileptogenesis. Neuropediatrics. 2017;48:5-18. doi:10.1055/s-0036-1597271

24. Caputo D, Iorio R, Vigevano F, Fusco L. Febrile infection-related epilepsy syndrome (FIRES) with super-refractory status epilepticus revealing autoimmune encephalitis due to GABAAR antibodies. Eur J Paediatr Neurol. 2018;22(1):182-185. doi:10.1016/j.ejpn.2017.11.005

25. Ismail FY, Kossoff EH. AERRPS, DESC, NORSE, FIRES: multilabeling or distinct epileptic entities? Epilepsia. 2011;52:e185-e189. doi:10.1111/j.1528-1167.2011.03293.x

26. El Sabbagh S, Lebre AS, Bahi-Buisson N, et al. Epileptic phenotypes in children with respiratory chain disorders. Epilepsia. 2010;51 (7):1225-1235. doi:10.1111/j.1528-1167.2009.02504.x

27. Bindoff LA. Mitochondrial function and pathology in status epilepticus. Epilepsia. 2011;52(Suppl 8):6-7. doi:10.1111/j.15281167.2011.03223.x

28. Appenzeller S, Helbig I, Stephani U, et al. Febrile infection-related epilepsy syndrome (FIRES) is not caused by SCN1A, POLG, PCDH19 mutations or rare copy number variations. Dev Med Child Neurol. 2012;54(12):1144-1148. doi:10.1111/j.1469-8749.2012.04435.x
29. Carranza D, Harvey AS, Iona X, et al. Febrile infection-related epilepsy syndrome is not caused by SCN1A mutations. Epilepsy Res. 2012;100:194-198. doi:10.1016/j.eplepsyres.2012.02.007

30. Nabbout R. Autoimmune and inflammatory epilepsies epilepsies caused by epilepsies with inflammation. Epilepsia. 2012;53:58-62. doi:10.1111/j.1528-1167.2012.03614.x

31. Wright S, Vincent A. Progress in autoimmune epileptic encephalitis. Current Opinion in Neurology. 2016:151-157. doi:10.1097/ WCO.00000000000000304

32. van Baalen A, Häusler M, Plecko-Startinig B, et al. Febrile infectionrelated epilepsy syndrome without detectable autoantibodies and response to immunotherapy: a case series and discussion of epileptogenesis in FIRES. Neuropediatrics. 2012;43(4):209-216. doi:10.1055/s-00321323848

33. Vezzani A, Rüegg S. Introduction to the 2nd meeting on Immunity and Inflammation in Epilepsy (IIE2016). Epilepsia. 2017;58(Suppl 3):7-10. doi:10.1111/epi.13780

34. Shorvon S, Ferlisi M. The treatment of super-refractory status epilepticus: a critical review of available therapies and a clinical treatment protocol. Brain. 2011;134(Pt 10):2802-2818. doi:10.1093/brain/awr215

35. Suleiman J, Brilot F, Lang B, Vincent A, Dale RC. Autoimmune epilepsy in children: case series and proposed guidelines for identification. Epilepsia. 2013;54(6):1036-1045. doi:10.1111/epi.12142

36. Sato Y, Numata-Uematsu Y, Uematsu M, et al. Acute encephalitis with refractory, repetitive partial seizures: pathological findings and a new therapeutic approach using tacrolimus. Brain Dev. 2016;38 (8):772-776. doi:10.1016/j.braindev.2016.02.006

37. Melvin JJ, Huntley Hardison H. Immunomodulatory treatments in epilepsy. Semin Pediatr Neurol. 2014;21(3):232-237. doi:10.1016/j. spen.2014.08.001

38. Nosadini M, Mohammad SS, Suppiej A, Sartori S, Dale RC, IVIG in Neurology Study Group. Intravenous immunoglobulin in paediatric neurology: safety, adherence to guidelines, and long-term outcome. Dev Med Child Neurol. 2016;58(11):1180-1192. doi:10.1111/dmcn.13159

39. Abend NS, Bearden D, Helbig I, et al. Status epilepticus and refractory status epilepticus management. Semin Pediatr Neurol. 2014;21:263-274. doi:10.1016/j.spen.2014.12.006

40. Bellante F, Legros B, Depondt C, Créteur J, Taccone FS, Gaspard N. Midazolam and thiopental for the treatment of refractory status epilepticus: a retrospective comparison of efficacy and safety. $J$ Neurol. 2016;263(4):799-806. doi:10.1007/s00415-016-8074-7

41. Fox K, Wells ME, Tennison M, Vaughn B. Febrile InfectionRelated Epilepsy Syndrome (FIRES): a literature review and case study. Neurodiagn J. 2017;57:224-233. doi:10.1080/21646821.2017.1355181

42. Nabbout R, Mazzuca M, Hubert P, et al. Efficacy of ketogenic diet in severe refractory status epilepticus initiating Fever Induced Refractory Epileptic Encephalopathy (FIRES) in school age children. Epilepsia. 2010;51:2033-2037. doi:10.1111/j.15281167.2010.02703.x

43. Appavu B, Vanatta L, Condie J, Kerrigan JF, Jarrar R. Ketogenic diet treatment for pediatric super-refractory status epilepticus. Seizure. 2016;41:62-65. doi:10.1016/j.seizure.2016.07.006

44. Gofshteyn JS, Wilfong A, Devinsky O, et al. Cannabidiol as a potential treatment for Febrile Infection-Related Epilepsy Syndrome (FIRES) in the acute and chronic phases. $J$ Child Neurol. 2017;32:35-40. doi:10.1177/0883073816669450

45. Kenney-Jung DL, Vezzani A, Kahoud RJ, et al. Febrile infectionrelated epilepsy syndrome treated with anakinra. Ann Neurol. 2016;80(6):939-945. doi:10.1002/ana.24806

46. Tan WW, Chan DWS, Lee JH, Thomas T, Menon AP, Chan YH. Use of magnesium sulfate infusion for the management of febrile illnessrelated epilepsy syndrome: a case series. Child Neurol Open. 2015;2. 2329048X14550067-Mar.

47. Zeiler FA, Zeiler KJ, Teitelbaum J, Gillman LM, West M. VNS for refractory status epilepticus. Epilepsy Res. 2015;112:100-113. doi:10.1016/j.eplepsyres.2015.02.014 
48. Gedela S, Sitwat B, Welch WP, Krafty RT, Sogawa Y. The effect of vagus nerve stimulator in controlling status epilepticus in children. Seizure. 2018;55:66-69. doi:10.1016/j.seizure.2018.01.010

49. Devinsky O, Cilio MR, Cross H, et al. Cannabidiol: pharmacology and potential therapeutic role in epilepsy and other neuropsychiatric disorders. Epilepsia. 2014;55(6):791-802. doi:10.1111/epi.12631

50. Devinsky O, Marsh E, Friedman D, et al. Cannabidiol in patients with treatment-resistant epilepsy: an open-label interventional trial. Lancet Neurol. 2016;15(3):270-278. doi:10.1016/S1474-4422(15) 00379-8
51. Baumeister FA, Oberhoffer R, Liebhaber GM, et al. Fatal propofol infusion syndrome in association with ketogenic diet. Neuropediatrics. 2004;35:250-252. doi:10.1055/s-2004-820992

52. Tang-Wai R, Mailo J, Rosenblatt B. Breaking the cycle: a comparison between intravenous immunoglobulins and high dosage prednisone in the treatment of medically intractable epilepsy in children. Seizure. 2017;47:34-41. doi:10.1016/j.seizure.2017.03.004

53. Geva-Dayan K, Shorer Z, Menascu S, et al. Immunoglobulin treatment for severe childhood epilepsy. Pediatr Neurol. 2012;46(6):375381. doi:10.1016/j.pediatrneurol.2012.03.015

\section{Publish your work in this journal}

Neuropsychiatric Disease and Treatment is an international, peerreviewed journal of clinical therapeutics and pharmacology focusing on concise rapid reporting of clinical or pre-clinical studies on a range of neuropsychiatric and neurological disorders. This journal is indexed on PubMed Central, the 'PsycINFO' database and CAS, and is the official journal of The International Neuropsychiatric Association (INA). The manuscript management system is completely online and includes a very quick and fair peer-review system, which is all easy to use. Visit http://www.dovepress.com/testimonials.php to read real quotes from published authors. 